\title{
Metal Ultrasonic Delay Lines
}

\author{
Russell W. Mebs, John H. Darr, and John D. Grimsley
}

\begin{abstract}
A study was made of the applicability of a number of metals and alloys for thermally stable ultrasonic delay lines.

A preliminary investigation was made of different types of pressure holders and adhesives for use in crystal transducer attachments and of the influence of specimen length on attenuation for various metals and alloys. The effect of cold-work, annealing, and specimen cross section on attenuation was also determined for a representative isoelastic alloy.

Measurements of temperature variation of signal attenuation, distortion, and delay time on a number of assembled delay lines indicated that an isoelastic alloy employing overcured epoxy-resin crystal attachments gave best over-all transmission characteristics. No correlation was obtainable between strength and sound-transmission characteristics with various cemented joints.
\end{abstract}

\section{Introduction}

The ultrasonic delay line has come into wide use in recent years with the development of radar, computers, and other electronic devices $[1,2,3,4] .{ }^{1}$ It is a means for delaying a signal for a predetermined short period to be accurately reproduced for use at an appropriate later instant. The delay line consists of (a) a transducer for converting the electronic signal to a sound signal, and (b) a suitable medium of appropriate dimensions in contact with the transducer that will transmit the sound signal to a second iransducer at the opposite end of the medium, or permit its reflection back to the original transducer; it is then converted to a usable electric signal.

The most common materials now used for delay lines are quartz [1] and mercury [5, 6]. Water, glass, and magnesium alloys have also been used in experimental lines. Where acceleration or changes in temperature of the line may be involved, many of these materials are eliminated from use.

The Metallurgy Division of the National Bureau of Standards was asked by the Ordnance Electronics Division to assist in the search for a more suitable material for delay lines by studies of metals that might be useful for the purpose. Two specific requirements were that it should satisfactorily transmit $10-\mathrm{Mc}$ ultrasonic pulses at temperatures ranging from $-50^{\circ}$ to $+200^{\circ} \mathrm{C}$, and possess a negligible temperature coefficient of sound velocity over this temperature range.

\section{Elements of High-frequency Sound-Pulse Transmission in Delay Lines}

The factors to be considered in the selection of materials for delay lines are (a) the length of delay required, (b) the carrier frequency, pulse length, and pulse shape to be transmitted, (c) the allowable variations in delay time with acceleration or change of temperature to which the line may be submitted, (d) the permitted maximum amounts of pulse attenuation and distortion, and (e) the allowed

${ }_{1}^{1}$ Figures in brackets refer to the literature references at the end of this paper. variations in pulse attenuation and distortion with acceleration or change of temperature.

Only a general survey will be given of the basic theory of ultrasonic transmission as related to delay lines of $50 \mu \mathrm{sec}$ or less. More comprehensive treatments have been given elsewhere [2, 4, and 5].

\subsection{Attenuation}

The signals being considered are $10-\mathrm{Mc}$ waves of short pulse length. The attenuation or diminution of the signal in traveling through the delay line is determined by (a) the conversion in the transducers of the electric signal to a sound signal, and vice versa, (b) the efficiency of transfer of the sound signal between transducer and delay line, and (c) the loss in signal strength in passing through the line. Such attenuation is measured in decibels $(\mathrm{db})$ which can be calculated according to formula 1

$$
d b=20 \log _{10} \frac{A_{o}}{A_{I}},
$$

where $A_{O}$ and $A_{I}$ are the output and input signal strengths, respectively. The factors involved in the loss in energy due to conversion between the electric and sound signals in the transducer will not be treated in this discussion.

The efficiency of transmission of the sound wave from the crystal into the delay line, or vice versa, is determined by the intimacy of contact between the crystal and line and by the degree of matching of the acoustic impedances of the crystal and delay line materials. For purposes to be discussed later, the side of the crystal opposite the delay line may be held under pressure by an appropriate material; the acoustic impedance of such material will likewise affect the transmission efficiency of the sound wave between crystal and delay line $[2,4]$.

The attenuation losses within a metal delay line have been attributed to the elastic hysteresis, the Rayleigh sound scattering, and the sound-diffusion process. The loss due to elastic hysteresis is attributed to an absorption from the sound wave of the energy required for cyclic stressing of the material [7]. This loss is a significant part of the total loss 
only at relatively low frequencies in the kilocycle range, being generally proportional to the frequency, whereas the other losses increase much more rapidly.

Sound scattering and diffusion occur only in the megacycle range; they are approximately proportional to the fourth power of the frequency when the grain size of the metal used is somewhat smaller than the sound wavelength and approximately proportional to the square of the frequency when the grain size is somewhat greater than the sound wavelength $[8,9]$. Such scattering and diffusion are due to the differences of the elastic coefficients in adjacent grains of the metal in the direction of propagation of the sound wave.

In addition to the principal signal transmitted through the line, there may be echoes caused by reflection of a portion of the signal from line ends and crvstal surfaces. These are detected at a later time than the principal signal, and for the proper operation of the delay line in some applications, their amplitude should not exceed a small fraction of the principal signal.

\subsection{Distortion}

The pulses transmitted through the delay line may not only be attenuated but may also become distorted.

A primary cause of pulse distortion is the superposition upon a weak pulse transmitted directly through the line of additional signals due to portions of the original signal that have been previously scattered and then reflected or refracted to the receiving crystal over a path longer than the direct pulse. Other causes may be attributed to poor contact between crystal and delay line, to improper cutting of the crystals, or to improperly prepared delay lines.

\subsection{Effect of Temperature on Delay Time, Attenua- tion, and Distortion}

The velocity of sound within a delay line, and hence the delay time, are determined by the specific physical properties of the material used. The velocities for longitudinal and shear sound waves differ and are expressed as follows:

$$
\begin{gathered}
v_{\text {long }}=\sqrt{\frac{E}{\rho}}=\sqrt{\frac{\lambda+2 G}{\rho}} \\
v_{\text {shear }}=\sqrt{\frac{G}{\rho}}
\end{gathered}
$$

where $E$ is Young's modulus, $\lambda$ is the bulk modulus of elasticity, $G$, the shear modulus, and $\rho$ the density of the material. The longitudinal velocity therefore is greater than the shear velocity.

In the present series of tests, $\mathrm{AT}$ and $\mathrm{AC}$ cut quartz crystals were used as transducers, and hence only shear waves were produced by the sending crystal. Furthermore, the directly transmitted portion of the signal was normal to the receiving crystal so that there was no transformation to the compressional mode; such transformations are obtained only upon reflection at certain oblique angles.

In the present investigation, emphasis was placed on determining which materials would give the least change in delay time per unit length with change in temperature between $-50^{\circ}$ and $+200^{\circ} \mathrm{C}$. The delay time, $L_{D}$, of a delay line can be expressed as

$$
L_{D}=\frac{l}{v_{\text {shear }}},
$$

where $l$ is the physical length of the line. From eq (3)

$$
L_{D}=l \sqrt{\frac{\rho}{G}} .
$$

Recognizing that $l, \rho$, and $G$ are all temperaturedependent, and assuming isotropic expansion of a polycrystalline material, it can be shown

$$
\frac{1}{L_{D}} \frac{\partial L_{D}}{\partial T}+\frac{1}{2 l} \frac{\partial l}{\partial T}+\frac{1}{2 G} \frac{\partial G}{\partial T}=0 .
$$

The temperature coefficient of expansion $(1 / l)$ $(\partial l / \partial T)$ for most metals is relatively small, and for the alloys of iron and nickel accounts for less than 0.25 percent of the change in delay time over a $250^{\circ} \mathrm{C}$ range. Thus a satisfactory thermally stable material of this type would be one with nearly zero or small negative temperature coefficient of the modulus $(1 / G)(\partial G / \partial T)$ over the required temperature range.

Little study has previously been made of the influence of temperature change on pulse attenuation and distortion. For temperatures ranging up to $200^{\circ} \mathrm{C}$, little or no change occurs in the microstructure of many metals. Conversely, cemented crystal attachments may be quite susceptible to temperature change. Therefore, one might expect temperature variation in transducer loss due to change in the nature of the crystal contact with the delay line. In discussing the effect of temperature on attenuation and distortion, a differentiation will be made in this paper between that obtained within the delay line and that obtained at the crystal connections.

\section{Test Materials, Specimens, Procedures, and Apparatus}

\subsection{Materials}

The 14 metals and alloys investigated and their compositions and conditions are listed in table 1 .

The magnesium alloys were studied as metals known to have good sonic properties and were also employed in the buffer lines to be described later. The high-purity and commercial nickel, Invar, 32percent-nickel iron and 18:8 chromium-nickel steel were tested to ascertain whether any relationship existed between the nickel content and degree of signal attenuation and distortion obtained. The aluminum single crystal was prepared in the Bureau's 
TABLE 1. Chemical composition and condition of the materials used

\begin{tabular}{|c|c|c|c|c|c|c|c|c|c|c|c|}
\hline \multirow{2}{*}{ Designation } & \multicolumn{10}{|c|}{ Chemical composition, percentage by weight } & \multirow{2}{*}{ Condition } \\
\hline & $\mathrm{Fe}$ & $\mathrm{Ni}$ & $\mathrm{Cr}$ & Mo & $\mathrm{Ti}$ & $\mathrm{Al}$ & $\mathrm{Si}$ & $\mathrm{C}$ & Mn & Others & \\
\hline $\begin{array}{l}\text { FS-1 magnesium al- } \\
\text { loy, a }\end{array}$ & $0.005 \max \ldots$ & $0.005 \max _{-}$ & .... & -... & -...- & 2.7 & 0.3 & -..- & 0.3 & $\left\{\begin{array}{lr}\mathrm{b} . \mathrm{Mg} & 95.64 \\
\mathrm{Cu} & .05 \\
\mathrm{Zn} & 1.0\end{array}\right.$ & Extruded. \\
\hline $\mathrm{J}-1$ magnesium alloy a & $0.005 \max \ldots$ & $0.005 \max _{-}$. & .... & .... & -.... & 6.5 & .3 & -... & .2 & 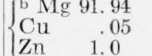 & Extruded. \\
\hline High-purity nickel ${ }^{\circ} \ldots$ & $0.032 \ldots$ & $99.70 \ldots$ & $\ldots$ & $\ldots$ & -... & -... & .10 & 0.01 & .03 & $\begin{cases}\mathrm{Cu} & .003 \\
\mathrm{~S} & 003\end{cases}$ & Annealed. \\
\hline $\begin{array}{l}\text { Commercial nickel..... } \\
\text { Invar d }\end{array}$ & $\begin{array}{l}0.10 \\
64.04 \mathrm{~b}_{-}\end{array}$ & $\begin{array}{l}99.49 \mathrm{~b} \\
35.96\end{array}$ & $-\cdots$ & -... & -... & $\cdots$ & - & .14 & .24 & $\mathrm{Cu} \quad .03$ & $\begin{array}{l}\text { Cold-drawn. } \\
\text { Reduced } 45 \text { percent in area } \\
\text { by cold-drawing. }\end{array}$ \\
\hline 32-percent-nickel iron ${ }^{\circ}$ - & $66.35 \mathrm{~b}$ & 31.95 & $-\cdots$ & $\cdots$ & $-\cdots$ & -... & .14 & .75 & .81 & & Reduced 51 percent in area \\
\hline $\begin{array}{l}18: 8 \mathrm{Cr}-\mathrm{Ni} \text { steel a } \\
\text { Carbon tool steel a } \mathrm{a} \\
\text { Aluminum.....- }\end{array}$ & $\begin{array}{l}72.86 \mathrm{~b}^{\mathrm{n}}-\mathrm{-} \\
98.4 \mathrm{~b}-\mathrm{-}\end{array}$ & $9.00 \ldots$ & $\begin{array}{r}18.00 \\
\cdots \\
\cdots-.\end{array}$ & $\begin{array}{l}-\cdots \\
\cdots-. .\end{array}$ & $\begin{array}{l}-\cdots \\
\cdots-- \\
\cdots-\end{array}$ & 99.99 & .20 & $\begin{array}{r}.14 \\
1.20 \\
-.\end{array}$ & .20 & 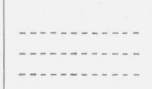 & $\begin{array}{l}\text { by cold-drawing. } \\
\text { Cold-drawn. } \\
\text { Annealed. } \\
\text { Single crystal grown by }\end{array}$ \\
\hline Isoelastic A d & 49.2 & $41.5 \ldots$ & $\ldots$ & 9.2 & $\ldots$ & -..- & $\ldots$ & $\ldots$ & .31 & $\ldots$ & $\begin{array}{l}\text { Bridgman method. } \\
\text { Reduced } 30.5 \text { percent in } \\
\text { area by cold-swaging; } \\
\text { annealed } 1 \text { hr. at } 500^{\circ} \text { C. }\end{array}$ \\
\hline Isoelastic B $0 \ldots$ & $52.8 \ldots$ & $36.7 \ldots$ & 6.8 & .56 & 0.22 & -... & .62 & .096 & .45 & .01 & Cold-drawn. \\
\hline Isoelastic $\mathrm{C}$ e $\ldots . . .$. & $53.4 \ldots$ & $37.0 \ldots$ & 7.0 & .06 & .18 & -... & .46 & .20 & .43 & $\left\{\begin{array}{l}\mathrm{Se} \\
\mathrm{W}\end{array}\right.$ & Hot-rolled. \\
\hline $\begin{array}{l}\text { Isoelastic } \mathrm{D}_{\mathrm{d}}^{\mathrm{d}} \\
\text { Isoelastic E } \mathrm{E}^{\mathrm{c}}\end{array}$ & $\begin{array}{l}47.6 \ldots \ldots \\
51.4 \ldots\end{array}$ & $\begin{array}{l}42.79 \ldots \\
35.6 \ldots\end{array}$ & $\begin{array}{l}5.97 \\
11.1\end{array}$ & -.. & 1.87 & $\begin{array}{c}.01 \\
\ldots\end{array}$ & $\begin{array}{c}.53 \\
---\end{array}$ & .11 & 17 & $\begin{array}{ll}\mathrm{Mg} & 18 \\
& \end{array}$ & $\begin{array}{l}\text { Hot-rolled. } \\
\text { Reduced approximately } 50 \\
\text { percent in area by cold- } \\
\text { drawing }\end{array}$ \\
\hline
\end{tabular}

a Type analysis. b By difference. o Determined by the Analytical Chemistry Section, National Bureau of Standards. d Determined by manufacturer.

Metallurgy Division. Of the isoelastic alloys, $\mathrm{A}$ was an experimental alloy [10]; B, C, and D were commercial alloys; and E was prepared and cold-rolled in the Metallurgy Division.

\subsection{Specimens}

Most tests were made on specimens approximately 6 in. long. However, other specimens ranging in physical length from $1 / 2$ in. to over 6 in. were tested; these permitted the measurement of the attenuation per unit length of a number of materials.

At the beginning of the investigation the specimens used were $0.35 \mathrm{in}$. in diameter over the central portion, and $1 / 2$ in. in diameter over a $1 / 2$-in. length at each end. Pressure holder compression fittings were used with this type specimen for holding quartz crystals directly against the line during test or for mounting cemented crystals before test. Later tests indicated that specimen cross section was of little consequence, providing it exceeded the transducer area. Hence specimens of various sizes were subsequently used, with a $1 / 2$-in. diameter the most common size.

A special jig was used for specimen preparation. It consisted of a steel cylinder with its two flat ends made effectively parallel by surface grinding. A square hole was carefully machined along its cylinder axis so as to be effectively normal to the end surfaces. Setscrews held the specimen in place during surface grinding and polishing (wet laps). The finished surfaces were flat and parallel within a few ten thousandths of an inch.

\subsection{Transducer Assembly}

One-quarter-inch square $\mathrm{AC}$ and $\mathrm{A} T$ cut crystals and $1 / 4$-in. round AT cut crystals were used in this investigation [11]. It was found necessary to check all crystals carefully as to uniformity of thickness (a 0.0001-in. variation was permissible) and to indicate the direction of the $X$-axis. Crystals were obtained with both faces silver-plated.

The compression fitting used for holding a quartz crystal against a line during a test consisted of a laminated plastic pressure holder containing a backing block and a socket-joint screw-tightening arrangement (fig. 1). The backing block was made of lead or steel, with the crystal facing surface being carefully polished. In some tests a 0.0005 -in. tinfoil layer was placed between the crystal and specimen. All facing surfaces were refinished between tests. A similar set of compression fittings made of aluminum were found to be unsatisfactory. They added excessive stray capacitance to the circuits.

The following adhesives were used in cementing crystals to the delay lines.

\begin{tabular}{|c|c|}
\hline Designation & Type of adhesive \\
\hline $\mathrm{AA}$ & $\begin{array}{l}\text { Elevated-temperature thermosetting } \\
\text { epoxy cement. }\end{array}$ \\
\hline $\mathrm{BB}$ & $\begin{array}{l}\text { Room- and elevated-temperature ther- } \\
\text { mosetting epoxy cement. }\end{array}$ \\
\hline $\mathrm{CC}$ & Silver paste. \\
\hline DD & Silicone. \\
\hline EE & Bakelite. \\
\hline FF & Beeswax. \\
\hline
\end{tabular}

All the adhesives except the beeswax were applied separately to line and crystal and allowed to dry at room temperature or in an oven before assembly. All connections were allowed to set under pressure; 


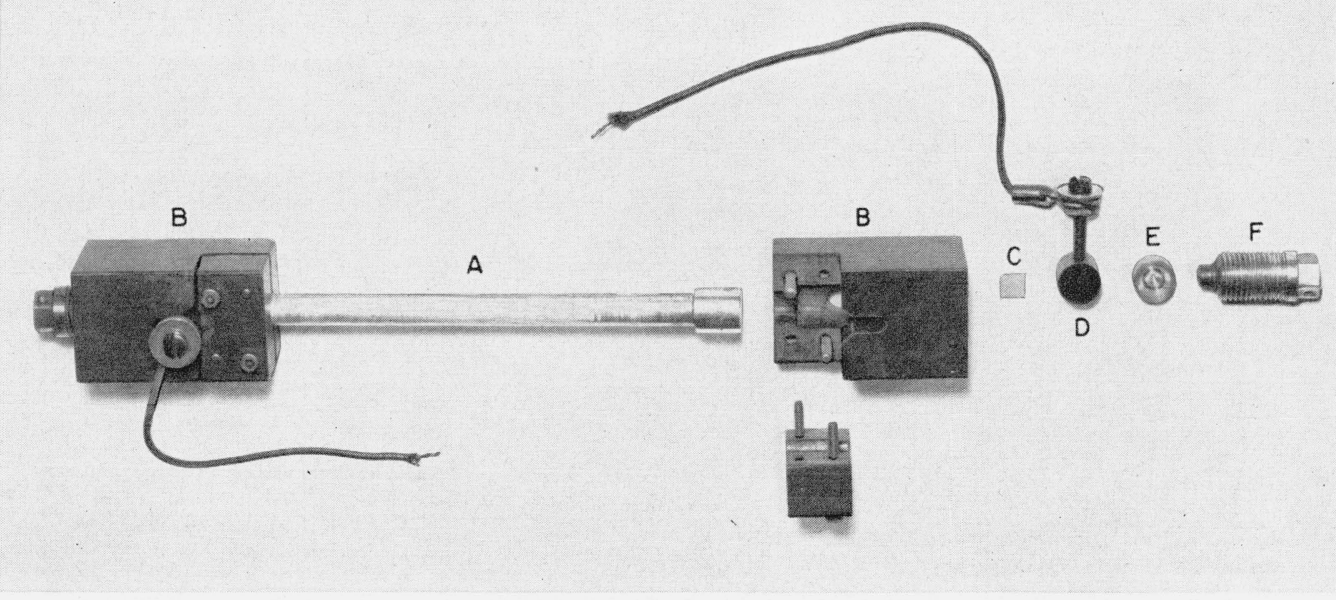

FIGURE 1. Bakelite compression fittings.

A, Specimen; B, Bakelite pressure holder; C, crystal; D, backing plate and electrical lead; E, socket plate; F, pressure screw with ball head.

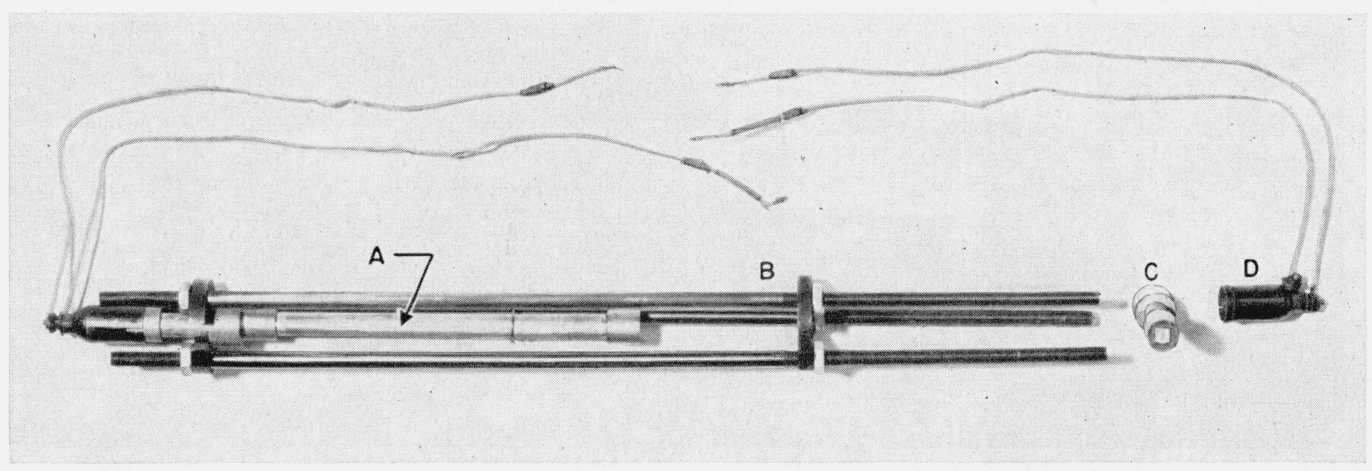

Figure 2. Buffer line assembly.

A, Specimen; B, clamp holder; C, magnesium-alloy buffer with cement attached crystal; D, shielded brass spring connector with electrical leads.

and additional curing was sometimes given the connection after the pressure was removed. Adhesive AA [12] was set at $170^{\circ} \mathrm{C}$ or higher before the removal of pressure. Adhesive BB showed a tendency to absorb moisture, which destroyed its soundtransmission qualities. Occasional baking out or storage of the assembly in a desiccator removed this trouble.

Attaching crystals to lines for each test by pressure holders or by cementing was time-consuming. Also after several tests with pressure holders a crystal usually fractured, and cemented crystals could not be salvaged for reuse. Furthermore, transmission efficiency varies with different crystals, and successive tests with compression fittings, using a single pair of crystals, was found to produce sufficient scatter of results to make difficult the measurement of attenuation per unit length on low-loss materials. Hence the buffer technique was developed and used in many tests made at room temperature. This is a modification of the method devised by McSkimin [13]. The two buffers consisted of FS-1 magnesium rods $1 \frac{1}{2}$ in. long. The $1 / 2$-in. length at each end was $1 / 2$ in. in diameter, and the central portion was $0.35 \mathrm{in}$. in diameter. Quartz crystals were cemented to one end of each buffer. By means of the special clamp shown in figure 2, the unmounted ends of the two buffers were held under pressure against a specimen of any desired length. It was necessary to lap the ends of the buffers adjacent to the test line between tests, and to regrind these ends whenever erratic test results indicated the need for this operation.

\subsection{Electronic Apparatus}

Figure 3 is a block diagram of the apparatus used for generating square-wave pulses and for measuring input and output signals for the delay line. A pulse generator capable of producing pulse lengths up to $10 \mu \mathrm{sec}$ and pulse repetition rates up to $5,000 \mathrm{c} / \mathrm{s}$ 


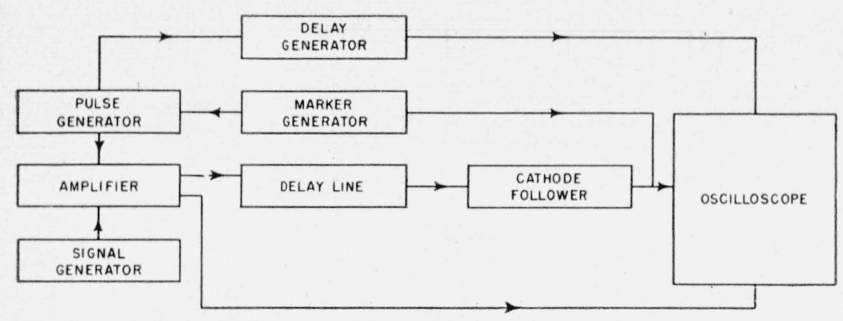

Figure 3. Diagram of test equipment.

triggered the output of a signal generator adjusted to produce a $10-\mathrm{Mc}$ constant-amplitude wave. The resultant pulsed $h f$ (high-frequency) waves were then amplified and fed into the input crystal transducer.

The hf voltage generated in the output crystal transducers was fed through the cathode follower into the cathode-ray oscilloscope where the amplified signal was placed on its vertical plates. The effective output impedence of the delay line is better matched by the input impedence of the cathode follower than by that of the oscilloscope. This permits a more efficient transfer of output energy to the oscilloscope. In some early tests, the cathode follower was not used; a much higher effective signal attenuation thereby was obtained (table 2).

The oscilloscope sweep applied to the horizonta! plates is triggered through the delay generator by the pulse generator; the delay generator can be adjusted to retard the sweep by as much as $1,000 \mu$ sec. The marker generator, which triggers the pulse generator, also produces small vertical markers every microsecond and larger markers every $10 \mu$ sec along the horizontal axis of the oscilloscope screen. An alternate circuit permits placing the input signal on the vertical plates. By accounting for the circuit amplification and attenuation of the various stages, the ratio of output to input signal can be measured by the oscilloscope. A Polaroid-Land camera was used for photographing these signal shapes.

\subsection{Measurements at Elevated and Low Tempera- tures}

A nichrome-wound alundum-tube furnace was used. This employed automatic control, providing a temperature that varied less than $5^{\circ} \mathrm{C}$ over the specimen length as measured by thermocouples attached to the center and ends of the specimen.

Some few tests were made with a shorter 5 -in. furnace in which 6 -in. test lines had their transducers extending beyond the ends of the furnace. Thus the temperature variation of transmission characteristics of the test material, exclusive of the transducers, could be obtained. All tests were made on specimens with cemented quartz crystals.

In order to obtain subzero temperatures, the test specimen was placed inside and midway as to the length of a thick-walled $1 \frac{1}{4}$-in. copper tube; dry ice was placed around this tube.

The rates of cooling after the application of dry ice and heating upon sublimation of the ice were sufficiently slow to enable accurate measurements of the temperature variation of input and output signal strengths and of delay time. A negligible temperature gradient was obtained along the specimen during these measurements.

\section{Effect of Type of Transducer Assembly on the Transmission of Ultrasound in Mag- nesium Alloy FS-1}

Table 2 lists the attenuation values obtained with a 6-in.-long FS-1 magnesium-alloy specimen, using pressure holders or cemented transducers. A torque wrench was used with the pressure holders and the torque adjusted to obtain minimum attenuation; fairly reproducible results were obtained by this method. It is apparent that the use of the cathode follower greatly decreased the attenuation attained.

TABLE 2. Attenuation at room temperature for various transducer connections on 6 -inch-long FS-1 magnesium-alloy lines

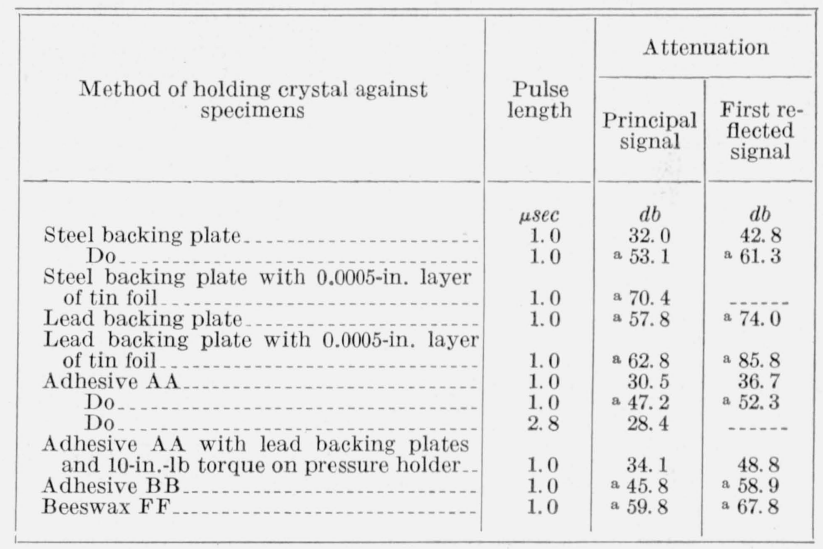

a These lines were tested with output connected directly to oscilloscope, remaining lines were tested with output connected through cathode follower to oscilloscope.

Examination of results indicates that lines cemented with adhesives $\mathrm{AA}$ or $\mathrm{BB}$ gave the lowest attenuation of the principal pulse. The use of beeswax FF as a crystal adhesive resulted in a fairly large attenuation. Although a fairly high attenuation was obtained with the lead-backed crystals when 0.0005 -in. tinfoil was inserted between crystal and line, successive echoes on this line were greatly diminished in comparison with the lines without the foil. The echoes obtained with the line cemented with adhesive AA were somewhat larger than those obtained with any combination of holder and backing plates. Furthermore, when a crystal cemented with adhesive AA was placed in a pressure holder and held with lead backing plates, the attenuation of the principal pulse was increased only a few decibels, whereas the first echo attenuation increased by about $12 \mathrm{db}$.

Of particular interest in these tests is the effect of transmission on wave shape. There are three general methods of evaluation of this effect, namely, (a) the visible change, as noted on the oscilloscope and which can be photographed; (b) the range of frequencies over which the amplitude of the principal 


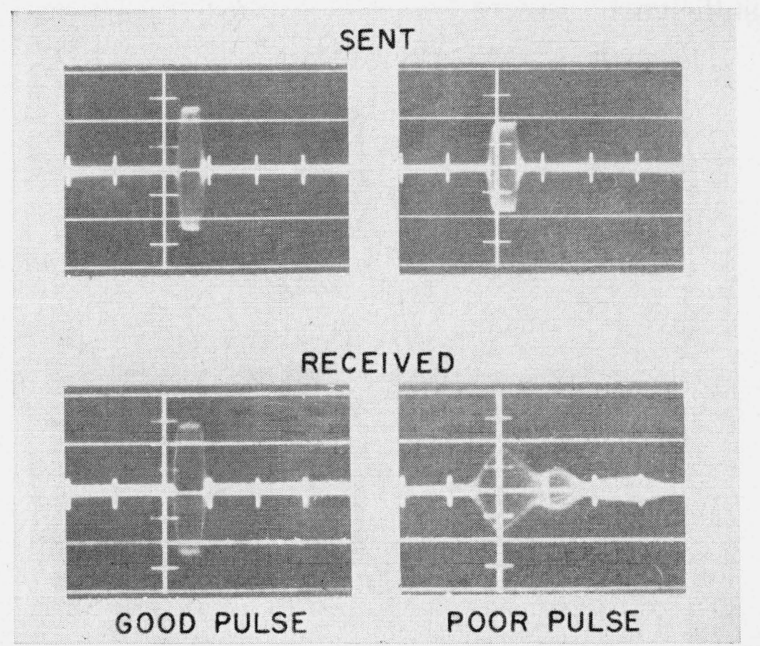

Figure 4. Representative oscillograms for sent and received pulses for good and poor pulse reproduction.

received pulse does not vary more than a fixed percentage of its maximum value for a given length of pulse; and (c) the minimum pulse length at which the maximum signal strength can be secured. Photographs of satisfactory and unsatisfactory reproductions of transmitted pulses are shown in figure 4.

Qualitatively, the use of lead backing plates on lines with tinfoil between crystal and line gave excellent reproduction. The same combination without tinfoil gave fairly good reproduction. The quality of reproduction was successively poorer in the steel-backed lines, the adhesive $\mathrm{AA}$ cemented lines, the adhesive BB cemented lines, and the beeswax FF cemented lines.

In addition to careful control of crystal quality, such factors as cementing technique or design of pressure holders and careful adjustment of pressure were studied in order to secure best sound transmission.

The efficiency of transmission of ultrasound by a given transducer assembly is also dependent on the structure, density, and other physical properties of the delay-line material as well as on the test temperature.

\section{Effect of Composition and Specimen Length on the Transmission of Ultrasound}

The buffer technique (fig. 2) was employed in this portion of the investigation. The pulse length employed was the minimum that would maintain maximum amplitude of the principal received pulse. This is hereafter referred to as the optimum pulse length.

Figure 5 shows the relationship between attenuation and length for the various materials investigated. The slope of each curve is a measure of the attenuation per unit length of that material. The intersection of each curve with the ordinate axis represents the total attenuation in the two buffers and in the interfaces between the buffers and the

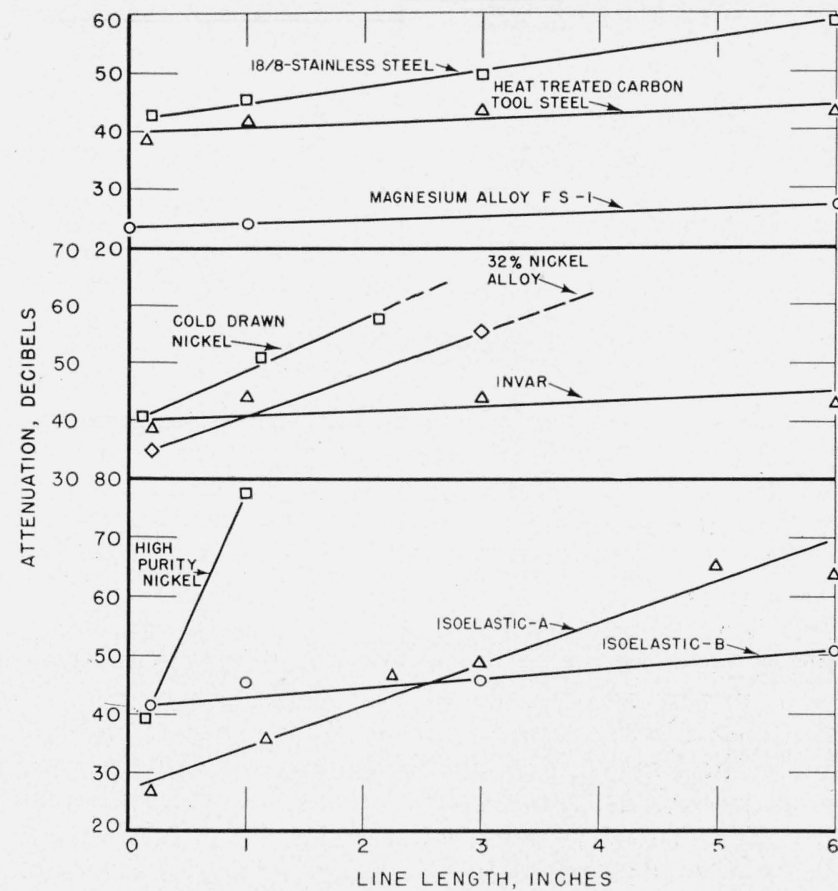

FIGURE 5. Variation of attenuation with line length for metals and alloys tested by buffer-technique method.

test material, and is referred to as the buffer loss. It is not the same for all materials due to differences in their physical properties and structure relative to that of the buffer material. Table 3 lists the buffer loss and the attenuation loss per inch length of a number of materials tested.

TABLE 3. Attenuation at room temperature of various materials as obtained by buffer-line technique

\begin{tabular}{|c|c|c|}
\hline \multirow{2}{*}{ Material } & \multicolumn{2}{|c|}{ Attenuation } \\
\hline & $\begin{array}{l}\text { Per inch } \\
\text { of length }\end{array}$ & Buffer \\
\hline $\begin{array}{l}\text { FS-1 magnesium alloy } \\
\text { Annealed high-purity nickel } \\
\text { Cold-drawn commercial nickel } \\
\text { Invar } \\
\text { 32-percent-nickel iron } \\
\text { 18:8 Cr-Ni steel } \\
\text { Spheroidized carbon tool steel } \\
\text { Quenches and tempered carbon tool } \\
\text { steel } \\
\text { Isoelastic A } \\
\text { Isoelastic B } \\
\text { Isoelastic C } \\
\text { Isoelastic D }\end{array}$ & $\begin{array}{l}d b \\
0.52 \\
44.0 \\
16.0 \\
0.86 \\
7.2 \\
2.8 \\
3.1\end{array}$ & $\begin{array}{l}d b \\
24.0 \\
33.0 \\
28.2 \\
40.0 \\
33.6 \\
42.0 \\
38.7 \\
39.8 \\
27.3 \\
41.2 \\
38.0 \\
35.2\end{array}$ \\
\hline
\end{tabular}

The quenched and tempered 1.2-percent-carbon tool steel and Invar provided values of attenuation per unit length similar to magnesium FS-1 alloy, though exhibiting higher buffer losses. Isoelastic alloy B and 18:8 $\mathrm{Cr}-\mathrm{Ni}$ steel gave greater values of attenuation per unit length with buffer losses comparable to the hardened tool steel and Invar. Commercial cold-drawn nickel, 32-percent-nickel iron, and isoelastic alloy A showed still greater values of attenuation per unit length and also exhibited buffer losses 

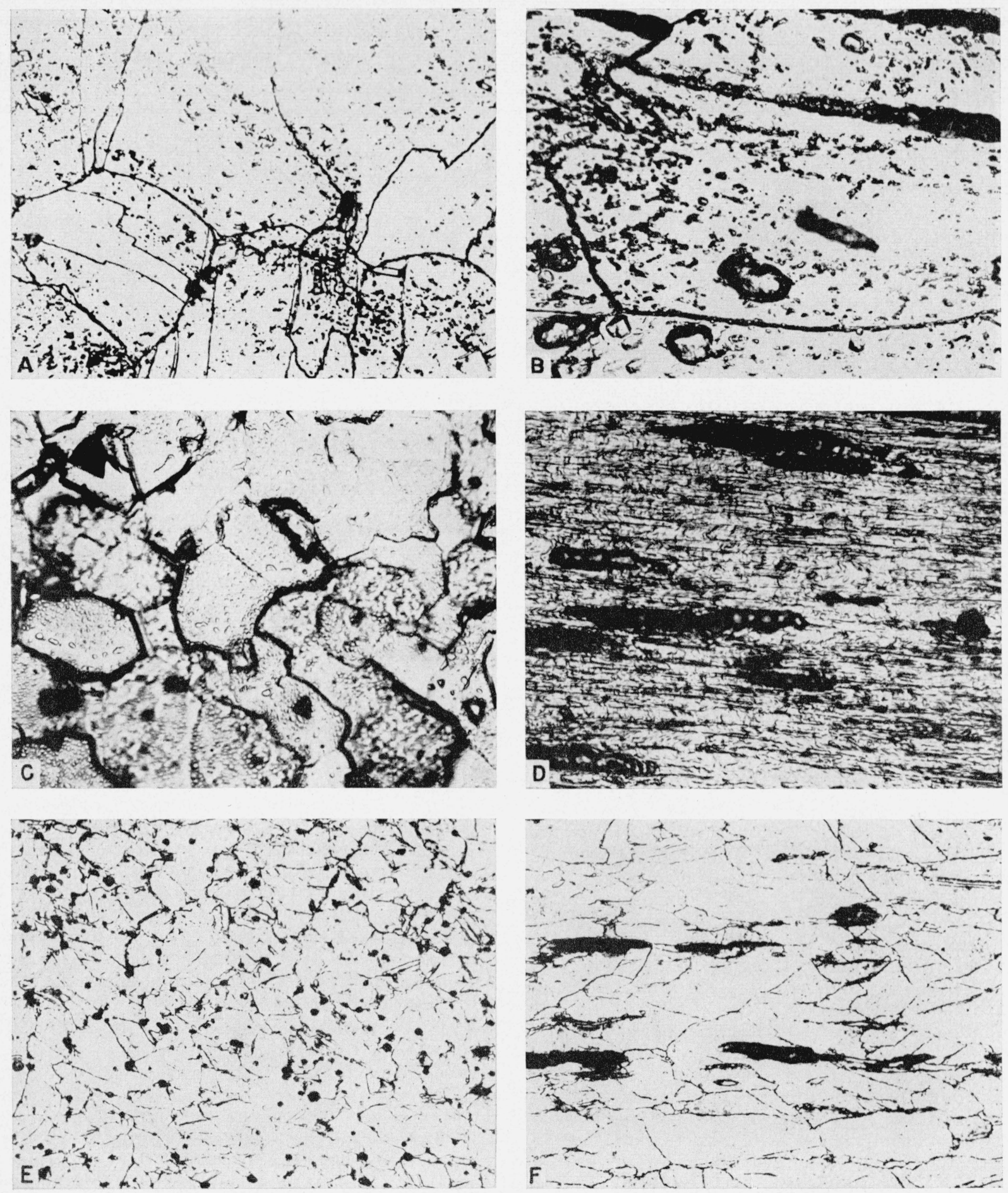

FIGURE 6. Micrographs of structure of various materials. Х500

A. Cold-rolled $36 \%$ nickel, $12 \%$ chromium, $52 \%$ iron alloy E, cross section, etched in Precision No. 2 reagent. The rather complex formula and method of preparing this reagent have been described in $[14]$. B. Cold-rolled $36 \%$ nickel, reagent. C. Annealed high-purity nickel, longitudinal section, etched in 6 parts of nitric (conc.) and 4 parts of glacial acetic acid. D. Cold-drawn isoelastic alloy $\mathrm{B}$, longitudinal section, etched in 1 part of ferric chloride, 10 parts of hydrochloric in Precision No. 2 reagent. F. Hot-rolled isoelastic alloy C, longitudinal section, etched in Precision No. 2 reagent. 
lower than Invar. High-purity nickel was the poorest transmitter of sound of the materials shown, although it did not show a correspondingly high value of buffer loss.

A short length of alloy $\mathrm{E}$ was found to give extremely high values of attenuation, as did the single crystal of aluminum. Attenuation per unit length could not be evaluated for these two materials.

A study of figure 5 and table 3 reveals that no general relationship exists between nickel content and attenuation per unit length for the respective metals and alloys. Factors such as other constituents, degree of cold work, and grain structure must also be considered as important. The isoelastic alloy $\mathrm{E}$ (fig. $6 \mathrm{~A}$ and $\mathrm{B}$ ) and the high-purity nickel (fig. 6, C) both possessed large grains, which may account, in part, for the high attenuation per unit length obtained. The very low attenuation per unit length of the quenched and tempered carbon tool steel can be attributed to its structure; when annealed, the tool steel was found to give a somewhat higher value. Of the commercial isoelastic alloys tested, alloy B was cold worked a greater amount, with the accompanying refinement of its structure (fig. 6, D), than was alloy $\mathrm{C}$ (fig. 6, $\mathrm{E}$ and $\mathrm{F}$ ). This, accordingly, might be presumed to be a prime factor for the low attenuation for alloy B. However, a few tests on another heat of hot-worked alloy $\mathrm{C}$ with a structure similar to that of figures $6, \mathrm{E}$ and $\mathrm{F}$, indicated this material to have as low attenuation per unit length as alloy B.

\section{Effect of Cold Deformation, Annealing Treatment, and Sound-Path Cross Section on the Attenuation of Ultrasound}

A rather extensive study was made of the ultrasonic properties of isoelastic alloy A during this investigation. This material was received in $1 / 4$-in.diameter size, having been cold-reduced 30.5 percent and stress-relief annealed at $500^{\circ} \mathrm{C}$ during manufacture. This material, as received, possessed a rather large attenuation per unit length and a low buffer loss, as shown in curve 1, figure 7 (also shown in fig. 5). Full annealing of this material tended to raise the attenuation length curve, as show in curve 2. When the as-received material was cold-swaged to 0.165 -in. diameter, that is, to a total cold reduction of 70 percent, an appreciable rise occurred in the attenuation values (curve 3 ). Stress-relief annealing of the swaged material caused a slight lowering of attenuation (curve 4).

In order to determine whether the increase of attenuation was due to the actual cold-working of the material or whether it was due to the reduction effective cross section of the sound path, some of the as-received material was machined to 0.165-in. diameter. Curve 5 for the machined material lies between curves 1 and 4 , and thus indicates that the rise in attenuation of the swaged material was partly due to the effect of cold-working and partly to reduction of cross section.

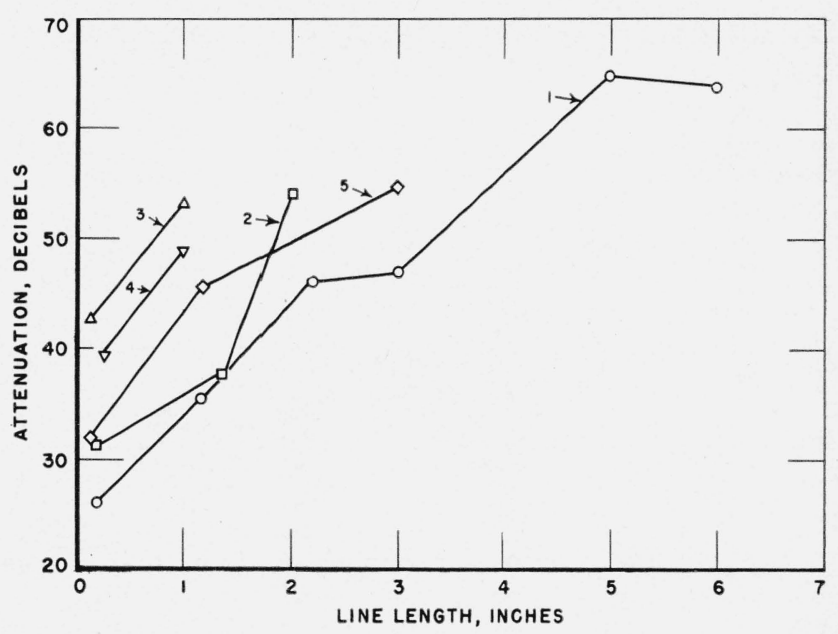

FIGURE 7. Influence of annealing treatment and additional cold-swaging on the attenuation-length characteristics of isoelastic alloy $A$.

Curve 1, as received (cold-swaged to $30.5 \%$ reduction of area and stress-relief annealed, 0.250 -in. diameter); curve 2 , fully annealed (0.250-in. diameter); curve 3 , additionally cold-swaged to a total of $70 \%$ reduction of area (0.165-in. diameter); diameter) plus stress-annealed at $400^{\circ} \mathrm{C}$ for 1 hour; curve 5 , as received, and machined to 0.165 -in. diameter.

It should be noted in this group of curves, however, that all except the curve for the annealed alloy possess nearly the same slope for the portion lying between zero and $1 \mathrm{in}$. in length, which is the extent of some of the upper curves. Thus the principal difference in attenuation values for these curves can be attributed to a difference in buffer loss. The principal effect of the additional cold-swaging, therefore, exclusive of the effect of reduction of cross section, was its apparent effect on the transmission characteristics of the buffer-specimen interface, i. e., the additional cold-working evidently tended to impede the transmission of sound between such surfaces. The increase in buffer loss by full annealing indicates that optimum sound-energy transfer occurred at the interfaces when this material had been only moderately cold-worked and stressrelief annealed (compare curves 1 and 2 ).

In the above tests, specimen diameters varied from 0.25 to 0.165 in., thereby affecting somewhat the sound-transmission efficiency, inasmuch as the effective sound-path cross section at the input crystal could be as great as $1 / 4 \mathrm{in}$. sq. Some tests were therefore made to determine the effect of increasing specimen cross section beyond that of the crystal on the sound-transmission efficiency. One-inch-long specimens of magnesium alloy $\mathrm{J}-1$ were machined to diameters ranging from $1 / 4$ to $1 / 2$ in. and tested between buffer lines. A maximum of only 3-db variation of attenuation was obtained in these tests, with no regular variation with diameter. Therefore, the effect of specimen cross section exceeding the crystal area evidently does not have a significant effect on the efficiency of sound transmission. 


\section{Effect of Temperature on the Attenuation of Ultrasonic Pulses in Various Materials}

Tests were conducted to determine the effect of temperature on both the delay-line material and the transducer connections attached to the delay line. Figures 8 and 9 show the variation of attenuation with temperature of various assembled delay lines. The pulse lengths are indicated in the legend of each figure. With the exception of curve 3 of figure 9 , the optimum pulse length was employed.

Figure 8 provides a comparison of various combinations of cements and curing conditions on 6 -in.long alloy $\mathrm{B}$ delay lines. In a preliminary test, employing the shorter 5 -in. furnace, with transducer connections extending outside the furnace, the attenuation was found to show negligible variation, except for a small decrease at $120^{\circ} \mathrm{C}$ (curve 1). Thus a temperature change of the delay-line material, in the range shown, caused little variation of attenuation - the transducer connection probably is the chief element affected. Later tests with the shorter

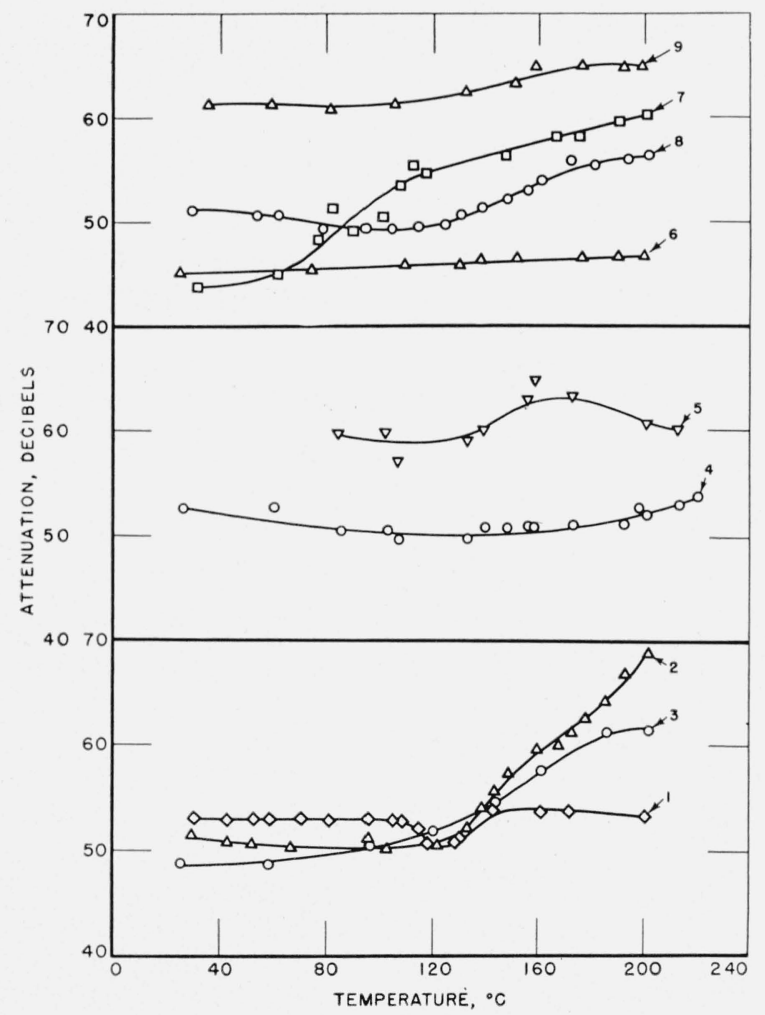

Figure 8. Influence of temperature on attenuation of 10 megacycle ultrasonic pulses in isoelastic alloy $B$, crystals cemented as indicated. Measurements made for initial received signal with entire line at indicated temperature, except curve 1.

Curve 1, 6-in. line, adhesive A A, cured $200^{\circ} \mathrm{C}$, tested in 5-in. furnace, optimum pulse length exceeds 10 microseconds; curve 2, 6-in. line, adhesive A A, cured $200^{\circ}$ pulse length exceeds 10 microseconds; curve 2 , 6 -in. line, adhesive AA, cured $200^{\circ}$ C, optimum pulse length 2.5 to 6.8 microseconds; curve 3,6 -in. line, adhesive A A,
cured $275^{\circ} \mathrm{C}$ after test 2 , optimum pulse length 2.75 to 5.4 microseconds; curve 4 , 5.3-in. line, adhesive BB, cured $280^{\circ} \mathrm{C}$, optimum pulse length 5.4 microseconds; 5.3-in. line, adhesive BB, cured $280^{\circ} \mathrm{C}$, optimum pulse length 5.4 microseconds;
curve 5, first reflection of test 4 ; curve 6, 5.3-in. line, adhesive $\mathrm{CC}$, cured $550^{\circ} \mathrm{C}$, curve 5, first reflection of test 4; curve $6,5.3$-in. line, adhesive CC, cured $550^{\circ} \mathrm{C}$,
optimum pulse length exceeds 10 microseconds; curve $7,5.3$-in. line, adhesive DD, optimum pulse length exceeds 10 microseconds; curve $7,5.3$-in. line, adhesive DD,
cured $250^{\circ} \mathrm{C}$, optimum pulse length exceeds 10 microseconds; curve $8,5.3$-in. line, adhesive DD, cured $350^{\circ} \mathrm{C}$ after test 7 , optimum pulse length exceeds 10 microseconds; curve 9, 5.3-in. line, adhesive EE, optimum pulse length exceeds 10 microseconds. furnace on a similar specimen, with cooling air blasts directed at the transducers, indicated a lowering of attenuation by as much as $8 \mathrm{db}$ at the higher temperatures. Such evidence suggests that the attenuation per unit length of the test alloy actually diminishes at higher temperatures.

The remaining curves in these figures were obtained with the entire delay line situated in the longer furnace. When adhesive $\mathrm{AA}$ was used with the recommended $200^{\circ} \mathrm{C}$ curing temperature, a sharp rise of attenuation (curve 2) occurred at elevated test temperatures in contrast to the less abrupt rise obtained after overcuring at $275^{\circ} \mathrm{C}$ (curve 3).

Curves 4 and 5, obtained with adhesive BB, cured at the optimum temperature ${ }^{2}$ of $280^{\circ} \mathrm{C}$ for this cement, show the variations of attenuation as obtained with the principal signal and the first reflection. Although a small temperature variation of attenuation is secured, the strong reflected signal and the large pulse length required for maximum signal are both deterring factors for satisfactory ultrasonic pulse transmission in such a delay line. Somewhat shorter optimum pulse lengths were obtained with adhesive AA (curves 2 and 3 ).

Curve 6 , obtained with silver-paste adhesive CC, indicates remarkably small variation of attenuation with temperature and a low level of attenuation, although requiring a long pulse length for optimum transmission.

Curves 7 and 8 , obtained with silicone adhesive DD, illustrate the flattening of the attenuation-

2 The optimum temperature for any cement is that minimum curing temperature resulting in the least temperature variation of attenuation.

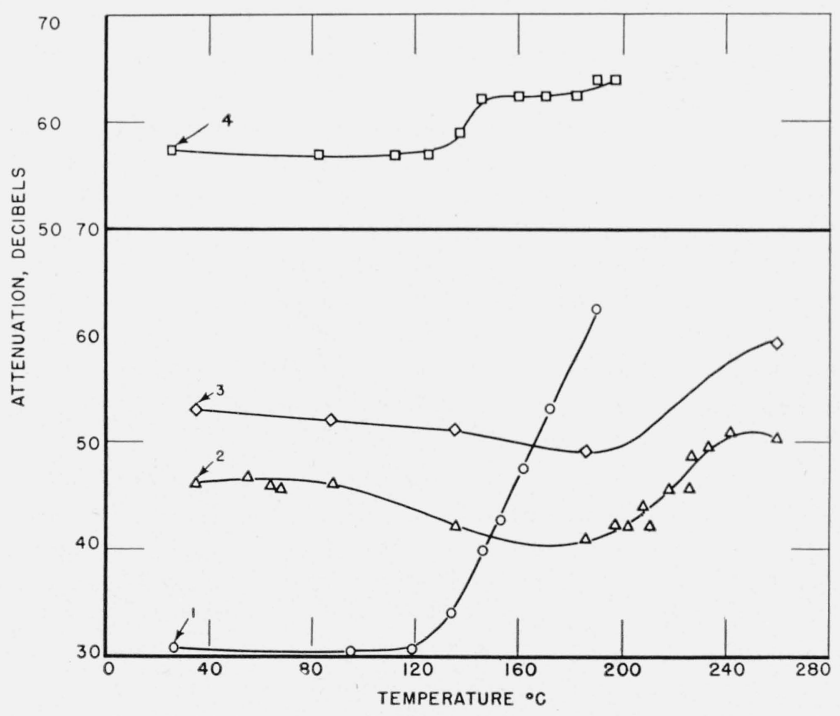

FIGURE 9. Influence of temperature on attenuation of 10 megacycle ultrasonic pulses of designated length in various delay lines, crystals cemented as indicated. Measurements made for initial received signal with entire line at indicated temperature.

Curve 1, 6-in. FS-1 magnesium-alloy line, adhesive AA, cured $200^{\circ} \mathrm{C}$, optimum pulse length 1 microsecond; curve 2, 6-in. FS-1 magnesiumalloy line, adhesive $\mathrm{BB}$, cured $200^{\circ} \mathrm{C}$, optimum pulse length exceeds 10 microseconds; curve 3 , same as test 2 with pulse length 3.7 microseconds; curve 4,6 -in. isoelastic-alloy C line, adhesive $\mathrm{BB}$, cured $200^{\circ} \mathrm{C}$, pulse varies 0.8 to 1.4 microseconds. 
temperature curve by elevating the curing temperature of this adhesive from $250^{\circ}$ to $350^{\circ} \mathrm{C}$. However, curve 8 lies somewhat above curve 6 and was obtained with a larger optimum pulse length than was curve 4 . The apparent plastic condition of silicone cements, even at elevated temperatures, obviously detracts from their usefulness for transducer connections in delay lines. Curve 9, for Bakelite cement $\mathrm{EE}$, indicates a fairly high attenuation with a low temperature variation. A fairly long pulse length is required.

Figure 9 shows the variation of attenuation with temperature for additional materials. Curves 1,2, and 3 were obtained with FS-1 magnesium alloy. Adhesive AA cured at $200^{\circ} \mathrm{C}$ (curve 1) provides a fairly short optimum pulse length, although producing a large variation of attenuation with temperature above $120^{\circ} \mathrm{C}$. Adhesive $\mathrm{BB}$, cured at $200^{\circ} \mathrm{C}$, gave a smaller variation of attenuation, although it had a somewhat higher room-temperature value. The rise in attenuation produced by shortening the pulse length from its optimum value of $10 \mu \mathrm{sec}$ (curve 2) to $3.7 \mu \mathrm{sec}$ is shown in curve 3. Data for these latter two curves were obtained during a single temperature-attenuation test. For this reason, curve 3 between the points at the two highest temperatures is drawn approximately parallel to curve 2 .

Curve 4 is for isoelastic alloy $\mathrm{C}$ with adhesive $\mathrm{BB}$. A small rise of attenuation occurs at about $150^{\circ} \mathrm{C}$.

Additional tests were made on other delay lines with similarly cemented crystals for temperatures ranging to as low as $-60^{\circ} \mathrm{C}$. Negligible variations in attenuation were noted below room temperature; these curves are not plotted.

It is evident from the above results that perhaps the principal variable in the efficacy of ultrasonic transmission in the isoelastic alloy delay lines is the transducer assembly. It was therefore considered of some interest to determine whether any relationship existed between the mechanical strength and soundtransmission efficiency of the cements for the various curing temperatures employed.

Some mechanical tests were made in which carbonsteel tensile specimens were cut in two, the cut surfaces being carefully polished and rejoined with the various adhesives by the same methods employed in attaching crystals to delay lines. These specimens were then tested in tension.

Maximum tensile strengths were obtained with joints of adhesives $\mathrm{AA}$ and $\mathrm{BB}$, when cured at $130^{\circ}$ and $150^{\circ} \mathrm{C}$, respectively. It will be recalled that the optimum curing temperatures for these cements for best signal transmission were $275^{\circ}$ and $280^{\circ} \mathrm{C}$, respectively. At these latter temperatures, the mechanical strengths of the joined bars were appreciably lower than maximum. It was also observed that silver-paste adhesive CC, which gave low signal attenuation, possessed quite low mechanical strength at all curing temperatures.

Evidently high strength and optimum signal transmission are not synonymous. The higher curing temperature, though probably causing a more brittle and weaker joint, may provide in such brittleness better signal transmission qualities. Thus the development of good transducer connections becomes purely empirical, inasmuch as the sound and mechanical properties of these elements cannot be correlated.

\section{Effect of Temperature on the Delay Time of Ultrasonic Pulses in Various Materials}

Figure 10 shows the influence of temperature on delay time, or time of transmission, of $10-\mathrm{Mc}$ ultrasonic pulses in assembled delay lines of various materials. Some of these tests were extended to subzero temperatures. The FS-1 magnesium-alloy line showed the greatest change in delay time with temperature. Of the isoelastic alloys tested, alloy C alone shows no variation in delay time up to the highest test temperature, $194^{\circ} \mathrm{C}$. The delay time for alloy $\mathrm{B}$ does not change up to $180^{\circ} \mathrm{C}$, but rises thereafter. The delay time for alloy $\mathrm{D}$ rises gradually with temperature up to $130^{\circ} \mathrm{C}$, above which it is nearly constant.

The sensitivity of measurement of delay time in these tests was approximately $0.1 \mu \mathrm{sec}$. For a $50-$ $\mu$ sec delay line, the limiting variation of the temperature coefficient of delay, measurable over a $200^{\circ} \mathrm{C}$ range is 10 parts per million. Thus for alloy $\mathrm{C}$, this coefficient evidently is 8 parts per million or smaller. By comparison, the temperature coefficient of delay for the FS-1 magnesium-alloy line averages about 380 parts per million over the test temperature range.

The small change in delay time above $180^{\circ} \mathrm{C} \mathrm{ob-}$ served with isoelastic alloy $\mathrm{B}$ may not be due to an actual change in properties of the alloy. A rather poor received-pulse shape was obtained with this material; it is possible that a change in pulse shape at the upper temperature levels due to some change

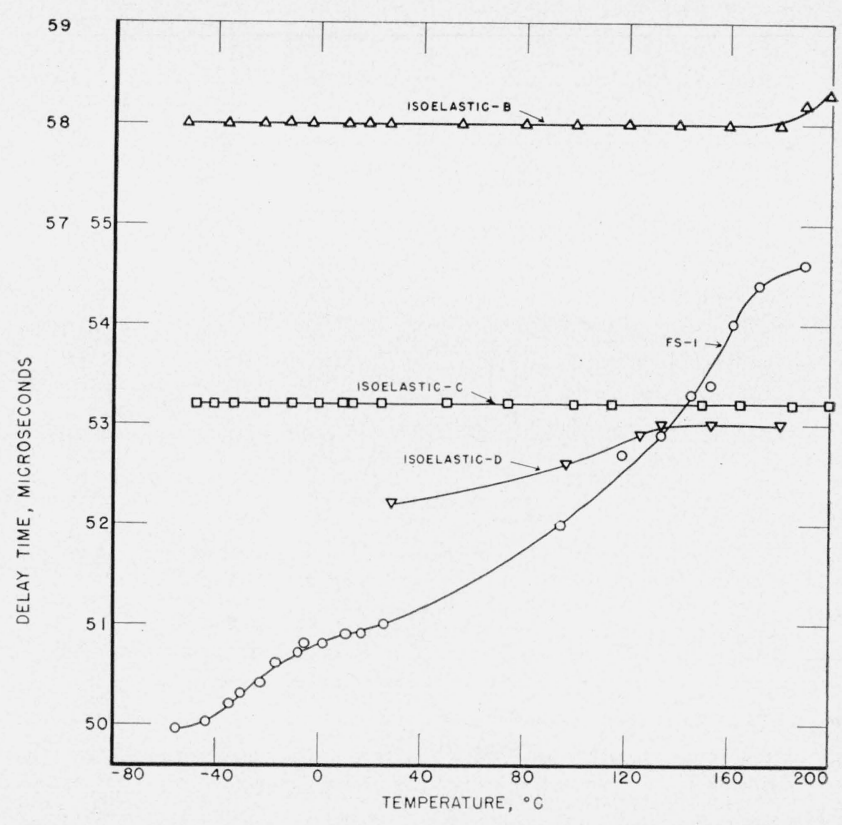

FIGURE 10. Influence of temperature on delay time for delay lines of various materials. 
in the properties of the adhesive might effect a shift in position of the received-pulse peak and thus effectively change the measured values of delay time.

\section{Delay-Line Characteristics}

The results of the above tests indicate limitations as to the optimum delay-line characteristics obtainable. Whereas a magnesium delay line may provide satisfactory attenuation and pulse-reproduction characteristics, its delay time varies appreciably with temperature. Constancy of delay time with low attenuation is most nearly fulfilled by isoelastic alloys $\mathrm{B}$ and $\mathrm{C}$; alloy $\mathrm{C}$ exhibits no measurable temperature variation of delay time. When crystals are attached to an isoelastic alloy $\mathrm{C}$ delay line with adhesive $\mathrm{BB}$ and cured at $200^{\circ} \mathrm{C}$, a reasonably high attenuation is secured (fig. 9, curve 5) that is nearly independent of temperature. In order to obtain a temperature invariant attenuation at a lower loss level, as typified by curves 4,6 , and 8 of figure 8 for isoelastic alloy $\mathrm{B}$, it is necessary to use delay lines that produce greater signal distortion and thus require a greater pulse length for optimum transmission. These tests would indicate that for these alloys a low attenuation is not compatible with a short optimum pulse length.

The number of variables that affect sound transmission make it difficult to provide a simple explanation of the occurrence of the above relationships. Some of these variables are (a) the damping of the crystals by the cements and delay line, (b) the effect of a poorly damped resonating crystal on signal distortion, (c) the relative acoustic impedances of crystals and line materials, and (d) the influence of the cement-layer characteristics on the sound transmission. Possibly other variables affect these relationships, necessitating an empirical study of each crystal, cement, and material combination in order to determine their suitability for use in delay lines.

These tests indicate that the transducer assembly is the greatest variable in obtaining satisfactory delay-line transmission over a wide temperature range.

A primary requirement is a thermally stable cement layer of suitable transmission characteristic. Even with an ideal cement, transmission is limited by the matching of the acoustic impedance of the crystal and delay line.

\section{Conclusions}

From measurements made of the transmission of $10-\mathrm{Mc}$ ultrasonic pulses in various metals and alloys, the following conclusions may be drawn:

1. The most efficient of the methods employed for conversion of electrical energy into sound energy within a delay line, and vice versa, is to attach quartz crystals to the delay line by an epoxy-type cement. The best reproduction of the principal transmitted signal wave shape, however, was obtained by use of laminated plastic pressure holders in which the quartz crystals were backed up with lead blocks and separated from the line by tinfoil.
This latter attachment method also produced the smallest reflected signals.

2. The attenuation per unit length of a material is best measured by the buffer test method. It varies from a very small value for the FS-1 magnesium alloy to very high values for annealed high-purity nickel and a single crystal of aluminum. The buffer loss ranges from small values for the FS-1 magnesium alloy to somewhat higher values for the isoelastic alloys, Invar, and 18:8 $\mathrm{Cr}-\mathrm{Ni}$ steel.

3. Although very coarse-grained materials presumably give a high attenuation loss, such losses do not show a fixed relationship with grain size for the finer structure commercial isoelastic alloys.

4. Although pure nickel possesses a high attenuation per unit length, there is no regular variation of attenuation with nickel content. Invar, a 36percent-nickel iron alloy was found to have an attenuation per unit length of the same magnitude as magnesium FS-1 alloy and quenched and tempered 1.2-percent-carbon tool steel.

5. In tests on the effect of cold-work and annealing on one of the isoelastic alloys, it was determined that the attenuation per unit length was not affected; however, the buffer loss was the lowest at an intermediate stage of cold-work.

The increase in buffer loss of the severely swaged isoelastic alloy was due only partly to the change in structure of the material, being also due partly to the reduction in cross section of the material. Where the cross-sectional area of the specimen exceeded that of the crystal, the effect of change of section could not be detected.

6. Of the various metals and alloys tested, two commercial isoelastic alloys, of different manufacture, were found to possess the least variation of delay time with temperature over the range $-50^{\circ}$ to $+200^{\circ} \mathrm{C}$. Both alloys contained approximately 36 percent of nickel, 7 to 8 percent of chromium, plus other minor constituents. The variation of over-all attenuation with temperature for these alloys was found to be due primarily to the transducer assembly employed; a small decrease of internal attenuation with rise in temperature was indicated on one of these materials. A negligible variation of attenuation with temperature up to $200^{\circ} \mathrm{C}$, at a low attenuation level, was secured by cementing the crystals to the more severely cold-worked of these two alloys with a silver-paste cement or one of the proprietary epoxy-type cements that had been overcured after attachment.

For the isoelastic alloys low attenuation was found to be incompatible with short optimum pulse length for any of the transducer connections tested. The limitations of obtaining good transmission apparently are associated with the characteristics of the cements and the relatively poor impedance match of quartz crystals with isoelastic alloys.

Somewhat higher curing temperatures were necessary for producing cemented joints having best transmission qualities, than for procuring maximum mechanical strength.

7. Although the buffer technique presents a rapid means for determining which metals possess a low 
attenuation per unit length or low transducer .losses at room temperature, the more tedious procedure of empirical determination of crystal, cement, and metal delay line combinations tested over the temperature range to which the delay line will be subjected, is necessary for satisfactory development of such lines.

Grateful acknowledgment is extended to W. M. A. Andersen of the Andersen Laboratories, Inc.; to M. E. Fine and W. C. Ellis of the Bell Telephone Laboratories; to W. A. Mudge and E. M. Wise of the International Nickel Co.; and to C. H. Fetter of the American Time Products, Inc., for technical advice and for furnishing some of the isoelastic alloys investigated. The authors are also indebted to W. E. Eberly, Carpenter Steel Co. for cold-drawing the 32-percent-nickel iron alloy and for furnishing the Invar alloy. Many thanks are due the following members of the staff of the National Bureau of Standards: L. T. Sogn, for providing much technical information on quartz crystals; T. E. Orem, for the aluminum single crystal; and R. H. Harwell, for preparing isoelastic alloy $\mathrm{E}$. The authors also acknowledge the extensive assistance and advice provided this work by E. Voznak of the Ordnance Electronics Division, the sponsoring agency.

\section{References}

[1] David L. Arenberg, Ultrasonic solid delay lines, J. Acous. Soc. Am. 20, 1 (1948).

[2] Crystal Research Laboratories Interim Engineering Reports, Contract W28-009 and W28-110 (July 1946 to December 1948).
[3] A. G. Emslie and R. L. McConnell, Radar system engineering, 1, chap. 16 (McGraw-Hill Book Co., Inc., New York, N. Y., 1947).

[4] H. B. Huntington, A. G. Engle, and V. W. Hughes, Ultrasonic delay lines, J. Franklin Inst. 245, 101 (1948).

[5] A. E. Benfield, A. G. Emslie, and H. B. Huntington, On the theory and performance of liquid delay lines, MIT Radiation Laboratory Report 792 (September 21, 1945).

[6] I. L. Auerbach, J. P. Eckert, R. F. Shaw, and C. B. Sheppard, Mercury delay line memory using a pulse rate of several megacycles, Proc. IRE 37, 855 (1949).

[7] R. L. Wegel and H. Walther, Internal dissipation in solids for small cyclic strains, Physics 6, 141 (1935).

[8] W. P. Mason and H. J. McSkimin, Attenuation and scattering of high frequency sound waves in metals and glasses, J. Acous. Soc. Am. 19, 464-473 (1947).

[9] W. P. Mason and H. J. McSkimin, Energy losses of sound waves in metals due to scattering and diffusion, J. Appl. Phys. 19, 940-46 (1948).

[10] M. E. Fine and W. C. Ellis, Thermal variation of Young's modulus in some Fe-Ni-Mo alloys, Trans. AIME 191, 761 (1941).

[11] W. P. Mason, Piezo-electric crystals and their application to ultrasonics, chap. XV (D. Van Nostrand Co., Inc., New York, N. Y., 1950).

[12] A new bonding resin, detailed results of laboratory tests on araldite, Sheet Metal Ind. 26, 1966-1988 (1949).

[13] H. J. McSkimin, Ultrasonic measurement technique, J. Acous. Soc. Am. 22, 413-18 (1950).

[14] Charles T. Flachbarth and Chester S. Pondo, Three new etchants for nonferrous metals, Metal Progr. 56, 688691 (1949).

Washington, June 8, 1953. 